REVUE HYBRIDE DE L'ÉDUCATION

Apprendre en et par un collectif dans l'instruction du travail éducatif

\title{
Accueil et orientation de jeunes mineurs délinquants placés en hébergement judiciaire
}

Auteur

Paul Olry

AGROSUP Dijon - UBFC, France, 


\section{REVUE HYBRIDE DE L'ÉDUCATION}

\section{Résumé}

Les régulations opérées en situation d'incertitude, bien documentées pour d'autres secteurs, sont mal connues dans les relations de service. Dès lors, comment les faire apprendre dans ces métiers ? Le travail éducatif, peu visible, s'apprend ainsi dans la durée. L'analyse de l'activité de travailleurs sociaux en foyer est étudiée en solidarité avec celle des jeunes mineurs délinquants qu'ils tentent de réorienter. Une étude ethnodidactique professionnelle qui rend compte de trois formes de régulation, liée à l'organisation (institutionnaliser les règles au sein d'un groupe de jeunes), au collectif (désaccorder son action) et aux individus (pragmatiser des concepts professionnels), est menée.

Mots clés : apprentissages professionnels ; activité ; collectif ; travail social 


\section{8}

\section{REVUE HYBRIDE DE L'ÉDUCATION}

\section{Problématique : apprendre en collectif de l'activité de chacun(-e)}

Dans de nombreux métiers existent des points aveugles sur « ce qui est à apprendre ». Les professionnels exécutent des tâches, mobilisent des procédés, s'engagent dans des processus divers, mais se trouvent parfois en difficulté pour énoncer ce qu'il faut savoir pour le faire. Il en est ainsi de nombreuses situations où "à force d'y aller, on finit par savoir le faire ». Souvent, les formations formelles ne sont pas d'un grand secours, car elles transmettent un discours sur ce qui est à faire, répercutent ce que les auteurs en disent, ce qui laisse les professionnels dans un embarras certain (Santelman, 2019).

Les courants francophones d'analyse clinique du travail proposent des cadres d'analyse en termes de cognition incarnée (l'explicitation, Vermersch, 1996), de construction de signes et de typification de contextes d'action (la démarche en cours d'action pour concevoir des formations, Guérin, 2012), de mobilisation subjective des sujets par et pour le métier (la clinique de l'activité, Clot, 2008) et de conceptualisation dans l'action pour cerner ce qui est à apprendre (cf. la didactique professionnelle, Pastré, 2011). Le travail échappe à ces analyses souvent pertinentes et complémentaires qui se centrent sur les sujets et leurs processus cognitifs ou conatifs. De ce fait, l'analyse partagée de ces points aveugles du travail par les opérateurs reste délicate. L'étude rapportée ici repose sur le principe d'allo-confrontations (Mollo et Falzon, 2004). L'analyse du travail ne résulte pas des seules verbalisations des professionnel(-le)s sur les images d'eux-mêmes en action via l'environnement de travail et les objets de l'action, mais du débat qui s'amorce avec un groupe de pairs rassemblé et étroitement associé aux descriptions, débats, faits, constats et processus, afin d'éclairer les impensés du métier.

Les objets de débat portent en effet sur l'identification d'équilibres qui rendent l'activité pertinente malgré les éléments de contrainte. La prescription de l'accueil par le juge contraint la décision d'accueil ; mais l'espace d'activité et la constitution interne du groupe de jeunes peuvent conduire à différer ou refuser l'admission ; les capacités des éducateurs enfin sont une variable à prendre en compte, notamment lorsque leur âge les rapproche des jeunes admis.

Dans des univers à prescription forte, ce type d'investigation est relativement fréquent (voir, par exemple, pour les ingénieurs du nucléaire, Jobert, 2011; Pastré, 2011). Dans des mondes professionnels à prescription faible, le défi qui intéresse les formations sur/par/à partir des situations de travail est celui posé par les entreprises à mission (Levillain, 2015). Parmi celles-ci, on étudie ici la Protection Judiciaire de la Jeunesse (PJJ) qui énonce ainsi sa mission : «La PJJ est chargée de mettre en œuvre les dispositions de l'Ordonnance du 2 février 1945 et ainsi d'assurer l'exécution et le suivi des mesures judiciaires prononcées à l'encontre des 


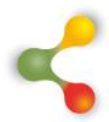

\section{REVUE HYBRIDE DE L'ÉDUCATION}

mineurs et jeunes majeurs par un magistrat ${ }^{1}$ ». II appartient « au terrain » d'y répondre. C'est pourquoi cette institution nous a demandé de contribuer à un groupe de pairs travailleurs sociaux afin de décrire, pour mieux la comprendre, leur activité pour suivre la prise en charge de jeunes mineurs délinquants. Ceux-ci (il n'y a là que des garçons) ont été interpellés en flagrant délit, déférés devant un juge des mineurs, leur âge leur offrant une dernière alternative à l'incarcération.

L'action des éducateurs PJJ est peu prescrite. Leur mission est de se mobiliser autour du jeune pour lui permettre de prendre ou de retrouver ses marques dans les différents domaines qui le concernent: famille, santé, scolarité, etc. Autrement dit, l'institution attend que chaque éducateur, chaque collectif de professionnels, au sein de lieux d'accueil "fermés " (ici des Centres de Placement Immédiat ou CPI), contribue au projet de stabilisation du jeune, reprenne son éducation (prise de conscience de sa situation et mobilisation pour s'insérer) et rende compte tant de son action que de ses résultats. Cette formulation globale peut s'exprimer plus prosaïquement par un but : «remettre sur les rails » les jeunes hébergés. Le pari de l'Institution est que parler avec un jeune de son orientation lui ouvrira " une possibilité de se situer lui-même pour un nouveau départ ». Ce but est poursuivi tout au long de leur présence dans la structure d'hébergement, au nom d'une prescription générale donnée aux travailleurs sociaux : faire en sorte que le jeune pense son « orientation de vie » en prélude à sa sortie de ce sas qu'est le foyer.

Cette prescription est peu relayée par la hiérarchie locale. L'action de cette dernière se résume à une mise à disposition de moyens pour faire le travail, sans organiser de coordination dans l'action de chacune et chacun. Le contrôle qui s'opère sur les travailleurs sociaux ne concerne pas le contenu du travail avec les jeunes, mais seulement à une conformité globale, ce qui n'exclut pas un appui ponctuel.

Or, dans les CPI, les travailleurs sociaux²(TS) sont régulièrement confrontés à des situations inattendues qui les déstabilisent dans la mesure où ils n'ont pas de solutions immédiates pour agir efficacement. Quelle activité ces TS déploient-ils, dans quel espace d'actions et au nom de quelles attentes?

\section{A/ La prescription de l'accueil des jeunes mineurs délinquants en Centre de Placement Immédiat (CPI)}

Les éducateurs (Éducateurs Protection Judiciaire de la Jeunesse EPJJ) ont pour mission d'assurer la prise en charge éducative d'un jeune

\footnotetext{
${ }^{1}$ Direction de la protection judiciaire de la jeunesse (France). (2021, 16 février). Dans Wikipédia.

https://fr.wikipedia.org/wiki/Direction_de_la_protection_judiciaire_de_la_jeunesse_(France)

${ }^{2}$ Dans les équipes, il y a des éducateurs, des moniteurs et en général un.e psychologue. Tous sont désignés comme "travailleurs sociaux».
} 


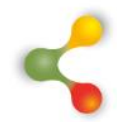

\section{REVUE HYBRIDE DE L'ÉDUCATION}

mineur confié par un juge, dans deux cas de figure : i) Le jeune est placé là en alternative à l'incarcération (prononcée à la suite d'une interpellation en flagrant délit) et dans l'attente d'un jugement ; ii) ce jeune est considéré en crise et présente un risque pour les autres ou pour lui-même (le placement est alors demandé pour prévenir ce risque).

L'accueil «immédiat » dans un centre d'hébergement constitue une activité complexe inscrite dans le temps suspendu d'un jeune, objet d'une ultime clémence de la décision de justice. Ce placement sous-entend, d'une part, un diagnostic d'état d'un jeune (l'expression en crise restant imprécise) à la suite de son arrestation; d'autre part, un choix du juge motivé par le risque de délitement lié à la détention, à prévenir. Dans une minorité de cas, le placement fait suite au signalement motivé d'un éducateur "de terrain » ('Action Éducative en Milieu Ouvert - AEMO) qui suggère au juge le placement du jeune avec l'accord de ses parents et le sien.

Cet accueil est discuté par l'équipe éducative du lieu d'accueil. Plusieurs cas de figure peuvent amener à sursoir à l'accueil : la présence dans le centre d'un jeune du même quartier, des critères d'éligibilité (ne pas venir directement d'un autre centre) et des motifs locaux (un groupe de jeunes stabilisé, dans une dynamique positive). Ainsi une marge d'appréciation est laissée à l'équipe d'éducateurs qui peut refuser la décision de placement du juge. Cette situation de prise de décision d'accueil est critique pour l'atteinte des objectifs fixés et pour l'équipe. Le terme générique «d'accueil » est en effet trompeur. La décision collectivement prise n'est pas qu'administrative. Pour les professionnel(le)s, elle vaut un engagement collectif auprès du jeune mineur accueilli de l'aider à se "stabiliser » et à conduire avec lui une action éducative finalisée par un projet d'orientation à construire.

\section{B/ L'espace d'activité : le foyer d'hébergement éducatif}

Cette mission s'exerce dans l'espace d'un centre d'hébergement. Mettre en sécurité un jeune et sécuriser son entourage se concrétise dans un placement en foyer. Ce placement est donc par nature transitoire : il est d'une durée de trois mois renouvelables, sans pouvoir excéder 6 mois, soit le délai donné à l'équipe pour réouvrir le champ des possibles d'une insertion. La décision judiciaire repose sur un espoir : que le jeune amende sa conduite au contact de l'équipe éducative et par un échange vertueux au sein d'un groupe de pairs.

Les EPJJ doivent faire du lieu de placement un «foyer d'hébergement éducatif ». Cette désignation exprime la volonté de passer d'une situation de crise d'un jeune, liée au délit commis, à une normalisation de son rapport à la loi dont il ignore souvent le contenu et les effets pour autrui et pour lui-même. La mission évoquée convoque plusieurs positions pour les éducateurs dans le dispositif : représentants de 


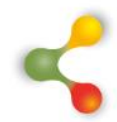

\section{REVUE HYBRIDE DE L'ÉDUCATION}

la loi et de la décision du juge, accompagnateurs du projet du jeune et substituts parentaux transitoires. Tous ne tiennent pas cette diversité de positions. Elles et ils se retrouvent autour d'un but : aider chaque jeune à passer d'un " équilibre rudimentaire à un équilibre supérieur " (Leblanc et Tessier, 1978). Ils s'accordent sur quelques points communs : enrôler le jeune dans un mouvement de prise de conscience de sa situation au regard des règles en société et formaliser avec lui des étapes par lesquelles retrouver une place sociale (apprenti, intérimaire par ex.).

\section{C/ L'activité de l'éducateur lors du processus d'accueil}

Toutefois, les prescriptions de l'emploi d'EPJJ ne permettent pas d'approcher l'activité des éducateurs dans le processus d'accueil. D'abord du fait de l'arrivée de ces jeunes mineurs délinquants : ils peuvent être déposés par la police, par un éducateur de milieu ouvert ou être récupérés dans le bureau du juge par un éducateur du centre. Ils peuvent être en sortie de garde à vue ou venir d'un autre centre (temporairement fermé).

À leur arrivée, les jeunes sont démunis matériellement (sans effets personnels), financièrement, voire psychologiquement. Durant ce moment délicat, postinterpellation et présentation au juge, l'arrivée dans le centre est perçue comme une rupture et une séparation pour ces jeunes.

C'est cette activité qui est l'objet de notre attention. Le processus « d'accueil » présente plusieurs phases : l'arrivée, l'admission et, au fil des jours, une sorte de pacification attendue du rapport du jeune à lui-même d'abord, à autrui ensuite (dont les éducateurs).

Durant l'arrivée par exemple, les éducateurs réalisent un certain nombre de tâches. Ils installent le jeune dans une chambre, lui présentent le groupe de pairs ainsi que les règles de vie dans le centre pour obtenir son acceptation formelle à les respecter. Ils surveillent particulièrement la première nuit et le premier dimanche, vécus par le nouvel arrivant qui sont autant de moments délicats, appelant leur vigilance.

Ainsi, le but dominant des éducateurs de la PJJ peut s'énoncer ainsi : organiser la prise en charge éducative d'un groupe de jeunes mineurs délinquants dans l'objectif que chacun prenne en main leur devenir en sortie de foyer (concrétisé dans un projet). Leur argument de conviction auprès des jeunes est que manifester un comportement social visant leur insertion, lisible par une juge, ne peut que les aider à "rester libres". L'administration de la justice entend en effet, l'orientation du jeune au sens d'un accompagnement contraint vers des solutions « standards » (retour à la scolarité, apprentissage d'un métier, etc.).

Pour l'équipe d'éducateurs, l'orientation de chaque jeune lui appartient. Le but de l'action n'est pas attaché à un seul jeune, mais au 


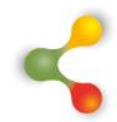

\section{REVUE HYBRIDE DE L'ÉDUCATION}

groupe de jeunes présents simultanément, dont il est indispensable d'assurer la paix interne.

Pour les jeunes présents dans le lieu de placement: i) l'action délictuelle est considérée d'abord par sa conséquence, soit se trouver en centre de placement ; ii) la recherche avec les travailleurs sociaux de pistes susceptibles de l'engager à réfléchir une autre " orientation » est difficile. Lorsque la justice pense une orientation référée aux espaces scolaire et professionnel, un jeune voleur de scooters y répond par la demande d'un CAP de réparations de motocycles; une autre rappelle que passer en prison «comme mon frère (...) donne un statut » de respectabilité dans la cité, le quartier.

Ainsi, le terme partagé d'orientation est l'objet d'investissements disjoints, dont le jeune est le point de rencontre. La (ré-) orientation d'un jeune est ainsi un «concept chaud» mobilisant émotions et affects des protagonistes de la prise en charge éducative. Pour le jeune, cela signifie s'extraire de l'univers qu'il connaît le mieux pour un « ailleurs social » dont il ignore les codes et dont il est jusqu'ici exclu. Pour les éducateurs, orienter, dans leur façon de faire le métier, signifie mettre en correspondance des caractéristiques d'un jeune avec celles attendues d'institutions pouvant soutenir son projet. C'est aussi réduire, pour le bien de ce jeune, le temps passé en foyer.

La question professionnelle que les éducateurs se posent est: comment amène-t-on un jeune délinquant à repenser une orientation qui l'a amené là? Cette prescription partagée en amène une seconde: comment ce mandat peut-il être travaillé collectivement?

En effet, le temps de séjour des jeunes (de trois à six mois) fait de leur orientation un défi, une mise en tension du travail collectif pour une équipe diversement rodée à l'action éducative auprès d'un tel groupe. Les façons de s'y prendre des éducateurs, tant avec chacun qu'avec le groupe de douze jeunes, soulignent les différences de points de vue des éducateurs à propos non seulement des projets d'orientation établis, mais également des façons d'exercer le métier. Autrement dit, le groupe de jeunes exploite toutes les différences, divergences de points de vue et d'action. Face à lui, pas de collectif d'éducateurs. D'objectif premier, l'orientation devient secondaire au regard de l'attention portée au groupe de jeunes par une perception rapide de signes et symptômes témoins d'évolutions internes afin de prévenir le risque de « horde ${ }^{3} »$.

L'activité des éducateurs traite de cette tension entre attention à chaque jeune et vigilance au groupe. C'est pourquoi on étudie d'une part ce qui s'énonce par l'analyse de l'activité lors de moments critiques

\footnotetext{
${ }^{3}$ L'accès au foyer nous fut une fois impossible, pompiers et gendarmes bloquant tous passages : le groupe avait tenté d'incendier le foyer.
} 


\section{$\&$}

\section{REVUE HYBRIDE DE L'ÉDUCATION}

(identifiés lors des échanges) où les travailleurs sociaux ressentent une impossibilité d'action. D'autre part, on s'intéresse à ce qui tente de s'apprendre collectivement au travers de décisions et règles collectivement adoptées, mais « oublieuses » des dilemmes quotidiennement rencontrés.

\section{Cadre théorique et conceptuel}

Ce qui précède souligne le caractère souvent «invisible » pour les gestionnaires de structure et le public de l'activité dans le travail social. Comment donc la partager entre professionnels ? Que révèle l'analyse de l'activité collective des éducateurs ? Que révèlent les modalités de travail du collectif du partage des conceptions de l'action éducative au sein de l'équipe pour le jeune? Que produisent ces modalités, conceptions et traces de l'activité en termes de formation informelle?

L'étude présentée n'a pas pour but immédiat de former les professionnels : il s'agit d'abord de comprendre, au travers de leur activité singulière, ce qui leur permet d'être efficaces ensemble. Si le travail est prescrit, des résultats souvent attendus de sa réalisation, les prescripteurs se préoccupent rarement des voies empruntées pour les atteindre. De même, les formations formelles, académiques ou (trop) proches du prescrit d'une situation de travail forment des travailleurs ajustés, mais peu adaptables. Inversement, une formation ignorante des situations de travail produirait quant à elle des généralistes, connaissant parfaitement ce qu'il faut faire, mais dans l'incapacité de le faire (Savoyant, 2008). La transmission professionnelle entre pairs peut dépasser cet écueil par l'échange sur la faisabilité des procédures formelles d'une part; d'autre part, les débats, les dilemmes, les controverses interrogent tant la possibilité que l'efficience du travaillé en collectif. Cette transmission tend à être instrumentée techniquement, qu'il s'agisse de simulateurs (VidalGomel, 2019), d'environnement virtuel d'apprentissage (Guidoni-Stoltz, 2020), etc. II s'agit moins de transmettre des savoirs que d'apprendre des situations (Pastré, op.cit.), de se développer professionnellement (Uwamariya ; Mukamurera, 2005).

En France, la place des autres, pairs, collègues, experts, pour apprendre, est l'objet d'une attention moindre tant l'individualisation est devenue un maître-mot des systèmes socioproductifs. Notre contribution questionne précisément l'intérêt de prendre appui sur des groupes de professionnels, pour aborder et apprendre de situations "critiques" auxquelles ces professionnels sont confrontés séparément. Selon Roy et Porchet (2017) : «les situations deviennent critiques lorsqu'elles cumulent une rupture ou une absence de solution de prise en charge et la mise en danger de la personne ou/et de son environnement »(p.340). C'est bien à de telles situations que sont confrontés les professionnels du travail social (Ferron et al., 2006). 


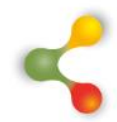

\section{REVUE HYBRIDE DE L'ÉDUCATION}

Cette recherche a ainsi pour but de décrire, sur la base des moments d'échange collectif, comment se constitue l'action de prise en charge par des travailleurs sociaux de jeunes mineurs délinquants placés en «Centre de Placement Immédiat » (CPI) en alternative à leur incarcération.

Nous postulons que l'échange de connaissances, le débat collectif et la réflexivité de ces professionnels, en miroir de leur action, constituent des moments d'apprentissages et de développement professionnel entre pairs.

Comme le suggèrent Mayen et al. (2015), l'activité ne s'étudie pas seulement «sur, dans ou en fonction » d'une situation, mais avec celle-ci. La situation est porteuse de caractéristiques agissantes avec lesquelles interagit l'action professionnelle. Par exemple, les conditions de travail constitutives de la situation agissent, préfigurent, contraignent cette action (p.112).

Notre question de recherche porte ainsi sur la conceptualisation collective dans l'action (Vergnaud, 1996) propre à des situations vécues ensemble. Au premier abord, on pourrait considérer que la compétence est collective parce que la conceptualisation des situations est partagée. Sur le constat que nos actes quotidiens actualisent une théorisation de la pratique, Vergnaud (1996) et Pastré (2011) promeuvent une théorie de la conceptualisation dans l'action. Les concepts produits ne sont d'abord pas scientifiques, mais pragmatiques. Pastré considère que l'organisation de toute activité est invariante pour une classe de situations donnée, et soutient une «image opérative» de l'action à conduire (2006), en complétude avec l'image cognitive qu'en propose la prescription.

Par quelle voie un groupe de pairs partagerait-il au-delà de l'action les conceptualisations produites à son endroit? La coprésence, la coopération des opérateurs ? La mobilisation de compétences individuelles éventuellement coordonnées? Autrement dit, nous formons des hypothèses sur l'élaboration collective des conceptualisations afférentes à la constitution d'un groupe de jeunes mineurs délinquants pour permettre une prise en charge efficiente de chacun d'eux.

\section{3 - Méthodologie}

\section{Le terrain}

Ces hypothèses et attentes ont été mises à l'épreuve au sein d'un dispositif agencé autour d'un noyau de deux fois 10 professionnels rapportant leurs réflexions à un collectif plus large. Ces professionnels, dits "éducateurs spécialisés " 4 travaillent par équipes de cinq, auxquelles il convient d'ajouter un cuisinier, une maîtresse de maison (chargée du

\footnotetext{
${ }^{4}$ Ces professionnels dans le système français sont titulaires d'un diplôme d'État.
} 


\section{$\&$}

\section{REVUE HYBRIDE DE L'ÉDUCATION}

ménage) et un veilleur de nuit. Le temps de travail est réparti en matin et après-midi dans ce foyer d'hébergement judiciaire d'urgence. En France, l'étude a été menée sur deux sites différents: l'un en région Lorraine, l'autre en banlieue parisienne. Les éléments rapportés ici concernent le site parisien.

\section{Le recueil de données}

Après une étude documentaire, l'étude a mobilisé deux modes d'investigation. Le premier, l'observation participante (Favret-Saada, 1977) a consisté (i) à "vivre » le quotidien de l'équipe d'éducateurs. Le but était de faire accepter la présence des chercheurs parmi les deux groupes de jeunes et d'éducateurs. Ces descriptions ethnographiques (i) révèlent des faits : les jeunes à priori soumis au règlement intérieur (par ex. sur les horaires) ne le respectent pas. Autrement dit, l'activité des éducateurs montre une difficulté à tenir les règles fixées collectivement dans plusieurs situations.

C'est pourquoi des observations focalisées ont été menées (ii) sur ces situations (accueil, réveil, repas, occupations,...) et les commentaires à leur endroit recueillis. Ces verbalisations (iii) mettent en exergue les ressorts de l'activité des éducateurs et amènent ces derniers à réinterroger les fondements de leur action. Ces temps réflexifs sur l'activité ouvrent des possibilités d'action, d'autres manières d'agir, à partir des réflexions adressées au groupe de pairs.

\section{Le traitement des données}

L'analyse de l'activité est menée collectivement sur la base d'une préanalyse du chercheur à partir d'une proposition de formulation des tâches redéfinies (Leplat, 1997). L'analyse seconde, menée en collectif avec le groupe de professionnels, fait apparaître des conceptualisations pragmatiques (Pastré, 2004). Le débat qui les révèle montre un ajustement des principes éducatifs, collectivement décidés par les éducateurs, aux contraintes de l'organisation collective avec les jeunes en situation.

Du point de vue du traitement des données, notre démarche s'appuie sur une approche psycho-ergonomique centrée sur un cadre conceptuel du travail collectif (Barthe et Queinnec, 1999). Ce cadre suggère que la coordination des actions individuelles opère au niveau des tâches, des ressources et des normes communes. La coordination constitue l'instrument de la coopération. Les données d'observations, comparant les professionnels, sont ainsi exploitées (Boccapege et Olry, 2015 pour le détail méthodologique) sous l'angle de la complexe genèse de cette coordination entre les acteurs.

C'est pourquoi les membres volontaires de l'équipe d'éducateurs ont été observés et suivis dans le cours de leurs journées de travail. Les 


\section{$\&$}

\section{REVUE HYBRIDE DE L'ÉDUCATION}

verbatims de leur intervention ont été consignés et soumis lors d'un moment ultérieur à des autoconfrontations ${ }^{5}$ simples (Pinsky et Theureau, 1987). L'ensemble observations/verbatims a été analysé sous l'angle des régimes d'engagement (Thévenot, 2000) fondé sur les coordinations au travail. Ce cadre est ici mobilisé face à la difficulté constatée des professionnels à élaborer un référentiel opératif commun (Chabaud et De Terssac, 1990) du but "orientation des jeunes mineurs ». L'engagement des éducateurs semble constamment pris à revers par les imprévus individuels et collectifs au sein du groupe de jeunes.

Ces constats ont amené les chercheurs à fixer l'attention sur les réunions où se construisent des décisions sur les règles que le collectif se donne pour tenir à la fois face et maintenir l'équilibre au sein du groupe de jeunes. La succession rapide de ces réunions révèle que la négociation entre professionnels des règles "à tenir ensemble », n'a d'égale que l'impossibilité à les tenir en situation « réelle ».

Les interprétations ont porté sur l'émergence, le maintien, d'un collectif dégageant un accord sur les buts poursuivis, en attente d'une action engagée par cet accord, mais en constante recherche d'une action coordonnée. L'interprétation s'est donc attachée à étudier ce qu'il en est pour l'équipe d'éducateurs observée. : comment s'agence ce qui fait collectif (référentiel opératif commun, cadre de références, etc.) et signe le métier, alors que l'action s'ajuste en permanence ?

\section{Principaux résultats}

Nous n'entrons pas ici dans le détail des résultats issus de 6 mois de présence dans ce $\mathrm{CPI}$. Nous présentons trois leviers de la régulation que l'activité collective mobilise. Le groupe de pairs est sous obligation de configurer collectivement des activités individuelles.

L'analyse ergonomique du travail a, dans ce cas précis, déjà été documentée par Augé (2000), qui a considéré le travail avec un jeune mineur délinquant sous l'angle des tâches à assurer qui procéduralise la prise de décision d'orientation (figure1). Pour autant, cette analyse de la tâche ne renseigne pas pour autant l'activité des professionnel(-le)s en termes de travail collectif autour de l'orientation d'un jeune.

\footnotetext{
${ }^{5}$ Siglées $A C^{\circ}$ plus loin dans le texte.
} 


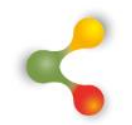

\section{REVUE HYBRIDE DE L'ÉDUCATION}

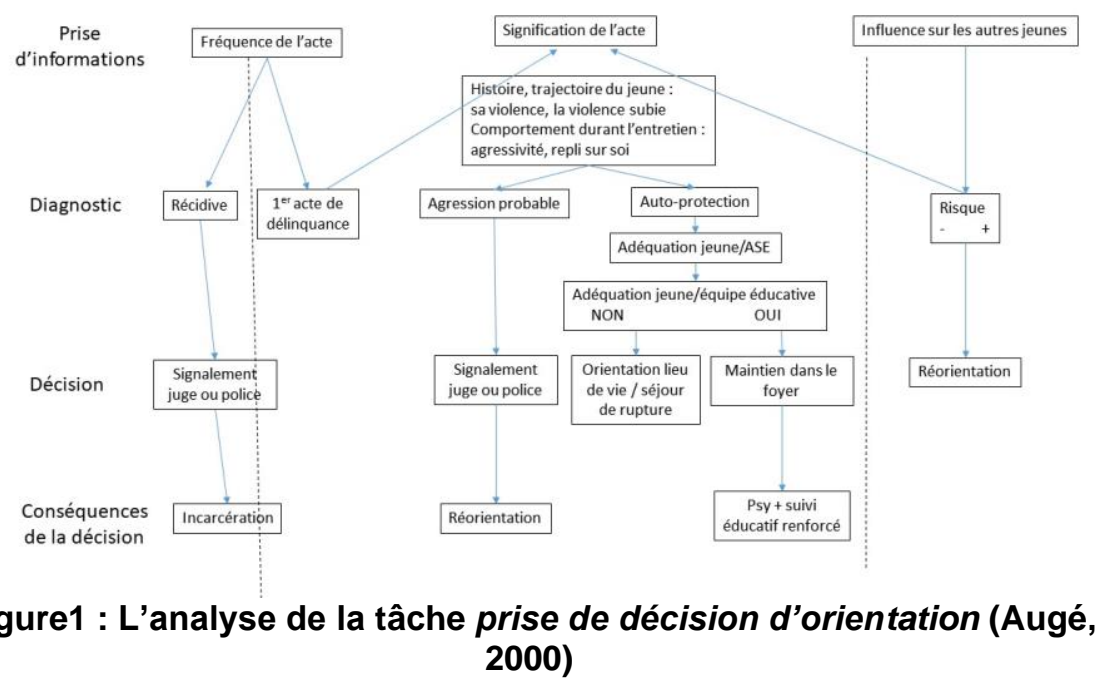

Le schéma ci-dessus nous retrace les étapes et les indicateurs qui déclenchent les diverses voies de la procédure. Cependant, il ne nous dit rien de l'enquête, des parcours et des indices, qui jalonnent ces étapes. Ce sont ces éléments de régulation que nous abordons maintenant en tant que leviers : organisationnel, collectif et individuel.

\section{Accueillir un jeune : une mise à l'épreuve de l'organisation}

Si toute demande d'accueil est formulée par un juge ou par une autre structure, elle est examinée en réunion dès lors qu'une place est disponible. L'avis de l'équipe est ici consultatif, le pouvoir discrétionnaire de l'équipe de direction s'exerçant s'il s'avère que sont mis en doute la capacité de l'équipe ou l'état du groupe de jeunes, à faire face à la perturbation que tout nouvel entrant ne manque pas de provoquer.

L'admission décidée (ou contrainte) d'un jeune s'ordonne selon un processus de travail idéal, voire idéalisé, comme ouvrage à chaque fois remis sur le métier si nous nous référons à la séance de travail rapportée ici. L'équipe a eu besoin de renommer, de formaliser à nouveau le format de tâches relevant de ce processus :

- l'accueil du MD, accompagné d'un parent/d'un policier, est effectué par un éducateur qui dispose du dossier de prise en charge ; s'en suit un premier entretien qui vise à informer le jeune (et sa famille, cas 2) des conditions d'accueil ;

- un livret d'accueil est fourni qui fixe les règles de vie au CPI auxquelles il est demandé au jeune de se conformer ; 


\section{8}

\section{REVUE HYBRIDE DE L'ÉDUCATION}

- un entretien est ensuite prévu dans la semaine avec la directrice ou le chef de service;

- un entretien avec la psychologue est également prévu.

Si ce processus semble parfaitement structuré et organisé, il constitue pourtant un processus idéal que chacun aimerait mettre en œuvre, mais qui se trouve plus ou moins respecté du fait des contraintes du service et aussi de la complexité de ces différents moments de travail à respecter par chaque membre de l'équipe. L'enjeu de l'équipe est de se rapprocher de cet idéal collectif.

Le collectif "prend» d'abord au travers de la forme d'une collaboration instituée de deux éducateurs référents (l'un principal, l'autre plutôt en remplacement). Désignés quelques jours après son arrivée, le choix de ces référents s'opère sur des critères permettant une répartition équitable des plans de charge des éducateurs et sur une première appréciation du comportement du jeune qui conduit à discriminer la «bonne personne » pour cette référence :

"Non attend, oh! Un môme comme ça, qui cherche toutes les occasions pour te mettre les mains partout, vaut mieux que ce soit toi (un collègue homme) qui t'le prenne »

À partir de la nomination du référent s'organise un processus visant la coopération (entendue comme activité contributive conjointe à l'atteinte du but commun) éducateurs référents - jeune, qui varie en fonction de la qualité d'interaction. Les autres éducateurs sont eux aussi invités à coopérer. L'accueil est ainsi une activité partagée qui place en observation le nouvel arrivant et documente ses comportements. Si le lexique pour qualifier chaque jeune est commun, de nombreuses divergences d'appréciation se manifestent.

Le collectif de métier se constitue donc au travers des débats de normes qui animent ces réunions. Ceux-ci se manifestent notamment en plusieurs occasions :

- en premier lieu, pour qualifier le rapport du jeune au cadre s'imposant à lui, selon sa capacité, sa disposition à être accueilli tant par les éducateurs que par ses pairs (certains jeunes réclament d'aller en prison, gage de prestige à la fin de peine) ;

- pour nommer ensuite, ce que l'espace domestique de la vie au foyer fait émerger d'attentes et d'exigences différentes, objets de désaccords et de conflits entre éducateurs (ex. : jusqu'à quelle heure autorise-t-on de regarder la télévision, de rentrer d'une sortie en ville, de manger dans sa chambre, de fumer à l'extérieur, etc.) ;

- pour formaliser le rapport d'évaluation qu'exige le suivi administratif et judiciaire du jeune, dont la rédaction est toujours délicate ; 


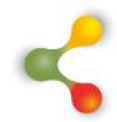

\section{REVUE HYBRIDE DE L'ÉDUCATION}

- $\quad$ pour crédibiliser un projet de fin de mesure, qui s'organise au titre de l'orientation en prévoyant des rendez-vous à l'extérieur, en organisant et planifiant des bilans réguliers avec le jeune, en faisant le lien avec sa famille, et en portant une attention plus particulière à sa situation matérielle (linges, affaires manquantes ou nouvelles...). Cependant, parmi les EPJJ, tous ne croient pas eux-mêmes au projet ainsi élaboré (par exemple, le projet de CAP horticulture de Ben ne convainc pas ses éducateurs référents qui connaissent sa passion unique des scooters qui lui vaut d'être là). Or, bien que le travail éducatif ne se fasse pas seulement avec les éducateurs référents, ceux-ci sont en première ligne pour en convaincre d'abord leurs collègues en réunissant les conditions d'un projet d'orientation crédible.

Le travail collectif de délibération qui encadre cette période délicate de l'accueil ne s'achève qu'au départ du jeune tant son attitude, ses avancées son comportement tout au long de sa présence dans le foyer sont un appel «constant » des interrogations, des peurs, des attitudes du jeune, sur lesquelles les éducateurs fondent leur travail. À ce titre, le collectif d'éducateurs opère des ajustements successifs qui recomposent les ingrédients du métier que sont les buts et conflits de buts, les normes qui orientent les règles d'action ainsi que les opérations pour qualifier l'état du groupe de jeunes et de ses membres.

À ce collectif d'éducateurs fait écho le collectif des jeunes présents dans le centre, parfois plus soudé que les professionnels, et qui conduit alors à un "rattrapage » de maîtrise des situations par l'équipe d'EPJJ. Tout se passe donc comme si l'activité éducative au sein de tels centres se jouait dans une capacité conjointe des collectifs de jeunes d'un côté, d'EPJJ de l'autre, de faire tenir (ou non) des conditions propices minimales à l'effectivité des prises en charge. Avoir du métier pour les EPJJ, c'est assurer une maîtrise de l'action éducative par et pour le collectif de professionnels par-delà les débats de normes qui l'anime.

\section{Instruire l'admission : une activité collective sollicitant les normes de métier des travailleurs sociaux}

Lors des réunions d'équipe, le temps consacré aux demandes d'admission est un moment éclairant. Les éducateurs ont des missions à remplir que les prescriptions peinent à décrire. L'admission dispose d'une procédure cohérente avec les normes de métier des EPJJ. En tant qu'éducateur, on se doit d'accueillir tout jeune mineur en détention provisoire. La procédure est la suivante :

- l'admission est acceptable et acceptée : le jeune correspond au profil ; - l'admission est refusée et argumentée, sur la présence d'un jeune de la même cité, de l'épuisement du droit à foyer d'hébergement, d'une irrégularité administrative ; il s'agit là d'argument d'éligibilité. 


\section{REVUE HYBRIDE DE L'ÉDUCATION}

Cependant, dans les faits retranscrits des réunions, l'admission est discutée sur d'autres plans du travail. Écoutons le groupe d'éducateurs PJJ dans ce moment de réunion où se discutent les admissions. Cet extrait des échanges lors d'une réunion où l'ensemble de l'équipe du foyer (incluant cuisinier et maîtresse de maison) conteste l'injonction à l'accueil :

Educatrice (E1) : Le père est décédé depuis ..euh depuis un certain nombre d'années déjà. II n'a pas eu de suivi psy, en tout cas ça n'apparaît pas dans la note. $X$ a été incarcéré l'année dernière pendant un mois, en... parce qu'il a été arrêté avec d'autres jeunes pour des vols...

E2 : violence en réunion....

$\mathrm{E} 1$ : violence réunion?

$\mathrm{E} 2$ : ils ne disent pas vol, ils disent violence en réunion...

E1 : la deuxième alors non ?... il y a deux incarcérations.

E2 : la deuxième c'est parce qu'il a violé son contrôle judiciaire (CJ)

E1 : (l'éducatrice hoche de la tête de manière affirmative) Oui c'est vrai... Donc la maman demande un placement pour ce jeune. Le jeune était complètement opposé au placement tant qu'il n'y avait pas de décision du juge de la placer. La juge ne s'était pas prononcé. Il y a eu donc une première incarcération d'un mois en octobre dernier. Lorsqu'il a été libéré, il a été placé dans une famille d'accueil en attendant d'avoir un Centre Educatif Renforcé ...II est resté très très peu de temps dans la famille d'accueil... Euh, le CER a été mis en place, mais finalement, il n'a pas respecté le cadre et finalement il a été incarcéré.

E3 : donc dans un CER, puis incarcéré

E4 : C'est ça la violation du CJ ?...

E1: Ouais!

E3 : ça a duré combien de temps le CER?

E1: Un mois!

E3 : Bon, il n'a pas eu vraiment le temps d'être évalué

E5 : Oui, mais ils ne les évaluent pas en CER!

Dir : oui, mais on dit que des fois les comptes-rendus des CER, c'est un ... ; en général on ne prend pas en sortie de CER! C'est...

E5 : oui, mais il peut y avoir une évaluation?

Dir : bref, il n‘a pas été évalué ce jeune?

E1: Non!

E6 : Il n'avait pas d'éducateur avant la première incarcération?

$\mathrm{E} 1$ : Si, il est suivi depuis ... euh 2019.

E6 : ben donc il y a eu une évaluation?

$\mathrm{E} 1$ : oui, mais une évaluation en foyer tu vois ! II n'est passé par un stade de...

E6 : non, mais l'évaluation, j'sais pas c'est une évaluation très progressive, insertion machin, etc.

E1. ce jeune habite Chelles et là, il y a une demande d'éloignement

Dir : mais on n'est pas une structure d'éloignement, on est un CPI! 


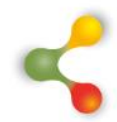

\section{REVUE HYBRIDE DE L'ÉDUCATION}

\author{
E1 : le jeune est dans la toute-puissance en famille. Là, il y a une audience \\ lundi... \\ Dir. Bon, on ne prend pas alors de toute façon, puisqu'il a fait CER...
}

Encadré 1 : Extrait d'une réunion d'admission - CPIP

La confrontation de quelques éducateurs aux images captées et aux notes prises de cet extrait de réunion révèle des raisonnements et arguments implicites dans l'échange. Ils relèvent d'une « pesée » en termes de risques et de capacité à y faire face. L'admission n'est pas séparée des risques de transgression du jeune mineur délinquant accueilli. La décision, a priori déléguée au groupe d'éducateurs (Augé, 2000), est le fruit de délicats arbitrages.

Ce jeune est très transgressif : "violences en réunion », rupture du contrôle judiciaire, non-respect du cadre du Centre Éducatif Fermé (CER), etc. Jamais le risque afférent n'est prononcé, mais l'argumentaire de refus s'annonce rapidement : «il n'a pas été évalué ». L'information collectée, imparfaite, n'autorise pas son accueil.

L'analyse de cette prise de décision souligne le rôle de la norme comme autre facteur non explicite de la décision. Le face à face avec le groupe de jeunes met la compétence des éducateurs à l'épreuve. Les procédures apprises, les discours sur l'action éducative et les règles élaborées lors des rencontres pour structurer l'intervention au sein du groupe de jeunes résistent mal aux contingences du quotidien qui contraignent l'action et la réflexion sur les situations.

Ainsi, ce qui s'avère en jeu dans le groupe d'éducateurs, c'est un débat sur les attentes de chacun. e. Quel respect des contraintes normatives de comportement est attendu de chaque jeune par chaque professionnel ? Quelle norme de réciprocité chaque professionnel mobiliset-il au travail avec les jeunes dont ils assurent la référence ? Quelle voie emprunte chaque professionnel (-le) pour faire respecter la contrainte normative dans ce lieu «protégé » qu'est le foyer d'hébergement?

Sans n'être jamais énoncé, le système d'attentes du groupe d'éducateurs est un déterminant de la décision d'admission. Alors que c'est la mission du foyer, le risque encouru à admettre un jeune instable dans le foyer, l'imprévisibilité des situations quotidiennes, la charge de référence que le nouvel arrivant appelle ainsi que les normes implicites du groupe de pairs conduisent au refus d'admission.

En témoigne ce verbatim d'une autre réunion d'admission : En réunion, on vient d'avoir un différend à propos d'un gamin : on n'était pas d'accord. La directrice a dit " on ne prend pas! On n'a pas la place ! »... Or, ce WE deux fugueurs et une mise à pied parmi nos jeunes! J'ai donc pris la décision d'accueillir un jeune. C'est une demande qui n'est pas une injonction. II 


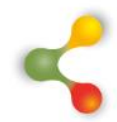

\section{REVUE HYBRIDE DE L'ÉDUCATION}

y a un coup de chaud au foyer de Vœu. Le môme était dans le cabinet du juge quand il a appelé, il fallait dire oui ou non. Il faut un peu de solidarité départementale.

Constatons que la prescription et les faits établis cèdent la place pour décider à la diversité des rapports à la norme éducative au sein de l'équipe. Ce qu'il en demeure, c'est la diversité des façons de faire, acceptables ou non, entre collègues, avec le groupe de jeunes. La recherche collective du commun, de l'invariance qui rassemble devient alors une clé pour tenir le métier (Clot, 2008).

\section{Orienter un jeune : une activité discursive quotidienne pour faire respecter les règles}

Si l'orientation est mythifiée par les prescripteurs sous forme d'un projet professionnel en bonne et due forme, elle est pour les éducateurs une organisatrice du parcours de chaque jeune vers sa sortie du foyer. C'est pourquoi l'activité d'orientation est d'abord diagnostique. Ce diagnostic opère pour trois protagonistes. Pour l'éducateur référent, le diagnostic porte sur le risque de cette prise en charge au regard de la qualité du travail ; vis-à-vis du jeune, le diagnostic s'effectue en tant que garant : il porte sur la possibilité et la faisabilité d'accompagner l'orientation du jeune, compte tenu du temps de placement et de l'équilibre du groupe qu'il intègre. Enfin, l'activité diagnostique engage en commun l'équipe d'éducateurs autour d'une projection d'un possible pour le jeune. À court terme, cela convie chaque professionnel à redéfinir pour soi-même l'exigence des tâches à réaliser au profit de ce jeune-là, dans le groupe de jeunes tel qu'il est, en tenant compte de la référence déjà assurée. L'analyse diagnostique conduit éventuellement à redistribuer les tâches entre collègues.

La discussion des faits et arguments susceptibles de faire pencher la décision d'admission ou non se poursuit une fois le jeune admis, car sa présence affecte des dimensions telles que l'état d'équilibre du groupe de jeunes. Là encore, cet équilibre est connu, mais peu "parlé ». Lors des échanges sont évoquées des situations relevant de temps clés: l'avant repas du soir, le dimanche soir, que nous avons observées, puis sont commentées par les éducateurs. Ces moments circonstanciés, parfois décrits dans le cahier du foyer, sont révélateurs de la redéfinition de leurs tâches par les éducateurs, tant du fait des règles établies en commun lors des réunions de service, que du contact dynamique en face à face avec un jeune ou avec le groupe de jeunes, ainsi que de l'acuité des problèmes qui se posent quotidiennement.

Si la norme oriente on l'a dit, la règle contraint. Le second travail qu'engagent les professionnels avec les jeunes concerne le respect de l'application des règles. Dans une première situation rapportée en séance, l'éducateur est de prime abord surpris par le départ de jeunes qui, à moins d'une heure du repas préparé, sonne comme un défi qui appelle une 


\section{REVUE HYBRIDE DE L'ÉDUCATION}

réplique de sa part. Celle-ci est construite autour d'un rappel à leur état en attente de jugement - à propos duquel il laisse sous-entendre qu'un comportement respectueux des règles peut leur éviter l'incarcération. L'extrait d'observation ci-dessous le rapporte.

\section{Situation 1 : la sortie d'un groupe de jeunes en journée}

\begin{tabular}{|c|c|c|c|}
\hline Tps & Eléments de l'activité & $\begin{array}{l}\text { Commentaires en auto- } \\
\text { confrontation (AC) }\end{array}$ & Etat de la situation \\
\hline $11 \mathrm{~h} 30$ & $\begin{array}{l}\text { " Allez faire un tour, OK! » } \\
\text { Les interpelle depuis la } \\
\text { fenêtre du } 1^{\text {er }} \text { étage : } \\
\text { E - « je vous rappelle que } \\
\text { le repas, c'est à } 12 \mathrm{~h} 30 \text { »! } \\
\mathrm{J} 1 \text { - non mais de toute } \\
\text { façon, on rentre ce soir! } \\
\mathrm{J} 2 \text { - de toute façon, ce } \\
\text { qu'on mange c'est pas } \\
\text { bon! On mange de la } \\
\text { merde ! On s'en va... } \\
\text { E - Je vous rappelle que } \\
\text { vous avez tous des } \\
\text { jugements bientôt! } \\
\text { J2- (d'en bas) : «tu ne vas } \\
\text { pas nous intimider? ? }\end{array}$ & $\begin{array}{l}\text { Le groupe se soude, } \\
\text { c'est inquiétant; } \\
\text { mais je n'ai pas à les } \\
\text { empêcher. Je leur } \\
\text { dois accueil, } \\
\text { surveillance, } \\
\text { protection, } \\
\text { éducation, c'est ce } \\
\text { qui est dit dans les } \\
\text { textes... } \\
\text {... bon, mais je vais } \\
\text { avertir les collègues } \\
\text { très vite. }\end{array}$ & $\begin{array}{l}\text { Alors que } \\
\text { l'éducateur du } \\
\text { matin passe les } \\
\text { coups de fil } \\
\text { nécessaires aux } \\
\text { prises des RV de } \\
\text { la semaine pour } \\
\text { chaque JMD, le } \\
\text { groupe de jeunes } \\
\text { au complet } \\
\text { signale qu'il sort. }\end{array}$ \\
\hline
\end{tabular}

Lors de la réunion d'orientation, l'argument porté par cet incident est celui du jugement de désynchronisation entre la rencontre à venir avec le juge et le comportement observé. Or, parallèlement, la réunion offre une possibilité de concevoir une synchronisation entre collègues des manières de faire pour prévenir l'action collective des jeunes, le constat étant établi que le groupe est en train de se souder puisqu'il sort « au complet ». 


\section{$\&$}

\section{REVUE HYBRIDE DE L'ÉDUCATION}

\section{Situation 2 : sortie d'un jeune faisant l'objet d'un contrôle judiciaire}

\begin{tabular}{|c|c|c|c|}
\hline Tps & Eléments de l'activité & Commentaires $\mathrm{AC}^{\circ}$ & $\begin{array}{l}\text { Etat de la } \\
\text { situation }\end{array}$ \\
\hline $11 \mathrm{~h} 40$ & $\begin{array}{l}\text { «D, tu t'en vas ?... Tu ne } \\
\text { veux pas rester avec } \\
\text { nous? } \\
\text { - Non! Je m'en vais, j'ai } \\
\text { juste oublié mes clopes! } \\
\text { - Tu sais que tu vois le } \\
\text { juge demain ? } \\
\text { - Moi j'assume... } \\
\text { - C'est pas le problème; } \\
\text { moi aussi j'assume ce } \\
\text { que je t'ai dit sur ce qui } \\
\text { peut t'arriver dès } \\
\text { demain. À toi de voir... } \\
\text { Est-ce que tu veux qu'on } \\
\text { sorte prendre un café } \\
\text { ensemble après le } \\
\text { repas? }\end{array}$ & $\begin{array}{l}\text { Là il venait chercher des } \\
\text { sous - il avait } 100 € \text { dans } \\
\text { sa chambre-. On ne sait } \\
\text { pas d'où ça vient, mais } \\
\text { on n'est pas armé pour } \\
\text { s'en occuper. } \\
\text { Mais il faut faire quelque } \\
\text { chose pour signaler } \\
\text { au(x) gamin(s) ce qui } \\
\text { dérape. } \\
\text { C'est pour ça que je joue } \\
1 / \text { une fonction de } \\
\text { rappel des alternatives } \\
\text { le concernant et que } 2 / \\
\text { je suis dans la } \\
\text { suppléance d'une parole } \\
\text { d'adulte qui sans moi } \\
\text { n'existe pas. }\end{array}$ & $\begin{array}{l}\text { Un jeune } \\
\text { signale qu'il } \\
\text { s'en va, avant } \\
\text { le déjeuner, } \\
\text { pour une } \\
\text { course rapide. } \\
\text { Il a interdiction } \\
\text { d'aller dans sa } \\
\text { cité dans } \\
\text { l'attente d'une } \\
\text { audience ou } \\
\text { d'un jugement. }\end{array}$ \\
\hline
\end{tabular}

Dans la seconde situation (2 mois plus tard), l'échange semble identique : l'éducateur réplique toujours, mais l'activité de réplique n'est pas seulement réduite à la joute verbale avec les jeunes, mais comporte un rappel à la règle et au cadre judiciaire de leur présence au centre. On peut enfin noter l'évolution de place de l'éducateur. Le premier extrait (situation 1) montre des interpellations réciproques: de sa position de référent, l'éducateur adopte une posture de répliquant (comme elle existe dans les défis entre rappeurs ou breakdancers). Dans la seconde situation, la réplique ne relève plus seulement de la joute. Elle témoigne d'une activité organisée. Comme le dit l'éducateur en autoconfrontation :

Avant je crois que je n'arrivais pas... j'ai toujours du mal... à ouvrir au-delà de ce que le jeune me disait. Je sais que quand on leur dit quelque chose, ils n'en ont rien à foutre, mais c'est pas pour ça qu'il faut du coup abandonner. En fait, on n'est pas seulement des référents institutionnels, il faut tenir sur la règle, même si c'est usant. Prendre la parole, répliquer, c'est aussi leur dire qu'on est là, leur garantir notre attention. Sinon le risque, il est tout pour eux! (éducateur $2, \mathrm{AC}^{\circ} 4$ ) 


\section{$\&$}

\section{REVUE HYBRIDE DE L'ÉDUCATION}

Dans ce propos, on reconnaît des concepts que fournit la formation des éducateurs pour penser leur action éducative et la place qu'ils y tiennent. Ainsi, la référence comme la réplique fait partie de ce cadre notionnel. Le rôle du référent est de travailler sur la globalité de la situation (jeune, famille, école, rééducations, partenaires...), de l'élaboration jusqu'à la rédaction des projets d'orientation. C'est cet argument qui est cette fois invoqué dans les débats lors d'une réunion de synthèse où les mesures et les jeunes concernés sont passés en revue.

Je trouve qu'il ne faut pas qu'on s'arrête au formalisme de la demande. Je trouve que c'est important d'être une équipe qui défend quelque chose par rapport à la Direction Départementale. On est déjà dans un mouvement général de prescription. Avant, on était dans des foyers de victimes avec quelques auteurs. Aujourd'hui on est une prison éducative, on a une mission de contention.

Et les gens se mettent au garde à vous. II n'y a pas si longtemps, quand la $D D$ disait quelque chose, tous les éducs se levaient et s'en allaient (...) On se demande qui fait la courte échelle aux mômes qui montent dans le charter.

Ainsi, au sein de la même équipe se distinguent chemin faisant les termes du débat. Le constat est établi que les jeunes enfreignent les règles, mais simultanément, un autre constat émerge : la procédure d'accueil et ses règles ne sont pas suivies par le collectif des éducateurs, au nom même de la référence (institutionnelle) d'une part et de la suppléance (actionnelle) d'autre part. Règles et normes collectives se trouvent interrogées : doit-on accueillir un jeune au titre de la procédure ou au titre de la nécessité dans laquelle il se trouve? En réunion de synthèse :

- $\quad$ Au quotidien, l'éducateur est garant de la continuité du suivi auprès du jeune et simultanément garant du travail engagé auprès de ses collègues, mais cette garantie ne peut être seulement affirmée auprès du jeune; elle doit s'incarner dans les propos de l'éducateur (E4).

- J'ai 24 ans et je suis une nana. Le dimanche soir, ils rentrent ensemble, bien chargés. Quand je leur dis de mettre la table et de venir manger... des fois je baisse les bras, je force pas. C'est difficile de s'imposer et on ne sait pas comment y faire seule (E5). 


\section{8}

\section{REVUE HYBRIDE DE L'ÉDUCATION}

- $\quad$ Oui elle a raison! et moi quand il y en a un qui rentre à $23 \mathrm{~h}$ (au lieu de $19 \mathrm{~h}$ ) et qui me dit «j'ai faim », et bien je vais avec lui faire un sandwich parce que je suis déjà contente qu'il soit rentré. (E8)

- $\quad$ Ah ben mois sûrement pas. S'il rentre à cette heure-là, c'est qu'il a mangé. (E1)

Les propos de ces éducateurs illustrent l'ambiguïté de la position du collectif d'éducateurs sur les règles à faire respecter. Établie en accord avec la prescription, celle-ci est constamment débordée par la capacité des pairs à faire face aux situations sur le moment. Ainsi, un éducateur chevronné, s'il comprend sa jeune collègue éducatrice, peinera à en tirer les conséquences en termes d'action collective et à se rendre disponible au-delà de ce à quoi il est tenu.

\section{Discussion : « faire métier en collectif ", dans et par les mises à} l'épreuve de l'activité

Le cadre de cette contribution invite à une réflexion sur les liens entre analyse de l'activité, individuelle, collective au sein d'une organisation dite "foyer» qui mêle institution et substitut familial. L'étude exploratoire présentée ici a proposé de saisir des concepts (suppléance, réplique, équilibre, référence, etc.) qui font ressource dans une situation en tant qu'organisateurs de l'action efficace (Pastré, 2011).

L'analyse de l'activité sur la base d'observations présentées lors de réunions confronte les éducateurs à l'image de leur action, sous forme de débriefing, provoque une réflexion sur l'action vécue et pas seulement réalisée.

Mises en travail entre éducateurs, les situations captées de face-àface avec les jeunes contribuent à construire une intelligibilité de la situation qui nourrit des conceptualisations en cours de ces situations.

L'organisation de l'action professionnelle est ici abordée sous l'angle des processus de pragmatisation et de conceptualisation (Pastré, 2011), qui mobilisent des objets, des états et des relations propres à la prise en charge éducative de jeunes mineurs délinquants. L'existence d'un schème de l'orientation post-placement se signale par un développement des concepts de réplique et de référence, sous l'effet du débat et des situations qu'il convoque.

Un deuxième aspect concerne la généralisation, c.-à-d. le traitement d'autres situations d'une même classe, par un concept organisateur 


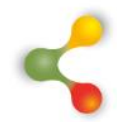

\section{REVUE HYBRIDE DE L'ÉDUCATION}

développé dans un autre cadre. C'est le cas du concept de référence. L'éducateur invoque dans son propos l'importance de ne pas cantonner la référence à sa fonction première de procédure institutionnelle. L'ensemble des tâches importantes que recouvre la référence sont mises à l'épreuve dans les situations de face-à-face complexes rencontrées par les éducateurs. Si la notion de référent est marquée par sa définition institutionnelle, elle est également marquée par son usage professionnel/individuel.

II suffit d'une réplique inadéquate pour rompre ce lien, avant tout institutionnel. À ce titre, on comprend que la réplique soit une tâche critique qui nécessite d'être pensée à tête reposée, alors que par nature elle est réaction à un événement, le plus souvent langagier. La réplique, de copie ou de reproduction du comportement du jeune, devient une réponse d'autrui adressée au jeune. On est ainsi fondé à donner un statut de concept pragmatique à la réplique, qui ne s'épuise ni dans une riposte, ni dans une réaction ou une répétition.

Enfin, le dernier aspect est celui de la stylisation de l'activité au regard du genre partagé par les professionnels. C'est en effet dans et par l'expérience que les individus développent des formes de conceptualisation opératoires et donc un style propre pour agir efficacement. II est dans cet exemple, ici inscrit dans une fonction de garant portée par l'éducateur, également indexé comme indice de la suppléance qu'il exerce en lieu et place des parents, mais aussi du juge. Cet autre but de la tâche, la suppléance, signale le lien entre la réplique et la garantie qu'elle représente pour le jeune.

Le développement professionnel de l'éducateur prendrait ainsi appui sur une conceptualisation progressive, par palier, comme cheminement au travers de quelques "vérités provisoires " nécessaires devant la grande abstraction, face au jeune, de l'action éducative. C'est un processus de généralisation qui, soutenant cette conceptualisation progressive, participe au développement professionnel de l'éducateur.

Nous avançons ici que les réunions d'admission sont aussi des moments d'apprentissage, dans la mesure où les débats qui s'y déroulent, portant sur les façons de faire, les règles et procédures de l'admission, conduisent l'équipe à instruire et décider collectivement dans des situations de complexité égale dont le périmètre s'accroît. Qu'y apporte alors le collectif au-delà des débats pour et à propos des jeunes ?

Ce qui « fait collectif », c'est d'abord le travail de débat au sens où l'équipe n'apparaît plus seulement réunie pour faire, mais comme instance d'interface, comme intercalaire social (Clot et al., 2000) entre les acteurs concernés. On y redéfinit la tâche, on précise les objets et on discute les opérations : comment est-ce que je me débrouille concrètement par rapport 


\section{$\&$}

\section{REVUE HYBRIDE DE L'ÉDUCATION}

à tel comportement ? Par-delà la décision prise, l'échange peut donner lieu à des redistributions de références, compte tenu des dynamiques en cours. De même, le travail collectif étudiant les conditions interroge dans le même mouvement la capacité et la disponibilité des pairs à œuvrer avec un jeune supplémentaire. Cela conduit à une meilleure synchronisation des tâches, des règles et de l'éthique pour l'action de chacun, mais également pour l'action collaborative entre les personnes.

Enfin, le travail collectif semble ouvrir une possibilité de débattre et de redéfinir les normes de l'admission d'un jeune, voire permettre un apprentissage. On retrouve ici la proposition de Clot (2008), selon laquelle le développement professionnel participe au développement personnel du sujet, qui naîtrait du mouvement entre le personnel (soi), le métier (transpersonnel), le genre (impersonnel) et l'interpersonnel (objet). C'est dans ce mouvement des instances que se produit du développement dont on ne peut opérationnaliser les critères sans méthodes et outils d'analyse de l'action.

L'activité, toujours professionnellement située, est un intégrateur des contextes et des situations : notre postulat est que son analyse permet d'en dénouer la chaîne et d'en révéler la trame. L'activité des éducateurs se caractérise par le fait d'avoir pour résultat d'agir sur autrui. À cet égard, décider (ou non) collectivement d'accueillir - incluant les deux volets d'accueil et d'orientation - constitue une situation qui, au-delà de sa singularité, est révélatrice de la complexité de l'action éducative.

Cette décision suppose un rappel des règles d'admission et donne lieu à des arguments relatifs à l'action organisée des sujets et aux transformations tentées et rapportées, dont nous faisons l'hypothèse qu'elles peuvent être considérées comme indicateurs d'une configuration collective des activités individuelles.

Si la gestion de la relation est le moyen quotidien essentiel pour l'éducateur de fixer son action auprès du jeune (soutenue par la connaissance d'un éventail de techniques d'action), le rapport aux règles commenté et décidé fonde ce qui rend son action légitime (et compétente ?) aux yeux de ses pairs. 


\section{REVUE HYBRIDE DE L'ÉDUCATION}

\section{Références}

Amade-Escot, C. et Venturini, P. (2009). Le milieu didactique : d'une étude empirique en contexte difficile à une réflexion sur le concept. Éducation et Didactique, 3(1), 7-43.

Augé, C. (2000). Interprétation et conceptualisation du cadre législatif par les professionnels de l'éducation spécialisée. L'exemple de situations de transgression [mémoire de maîtrise inédit]. CNAM.

Barbier, J-M. (1992). Évaluation de projets d'action et planification. Presses Universitaires de France.

Barthes, B. et Queinnec, Y. (1999). Terminologie et perspectives d'analyse du travail collectif en ergonomie. L'Année psychologique, 99(4), 663686.

Berton, F., Boru, J-J. et Barbier, J-M. (1995). Situations de travail, situations de formation. L'Harmattan.

Bernaud, J-L. (1999). L'évaluation les compétences individuelles en situation de travail. Revue Française de Gestion, nov-déc, 120-128.

Boboc, A. et Metzger, J-L. (2009). Du privé vers le professionnel, une dynamique des apprentissages croisés autour des TIC. Savoirs, 20(2), 158-179.

Boccapege, G. et Olry, P. (2015). Soutien à la parentalité : un double objet de conception pour le formateur. Education Permanente, (204), 1932.

Bourgeois, E. et Durand, M. (2012). Apprendre au travail. Presses Universitaires de France.

Clot, Y. (2008). Travail et pouvoir d'agir. Presses Universitaires de France.

Curie, J. (1998). Le discours de la compétence, ou l'expert et la diseuse de bonne aventure. Éducation permanente, (135), 133-142.

Dejours, C. (2003). L'évaluation du travail à l'épreuve du réel. INRA éditions.

Delgoulet, C., Boccara, V. et Santos, M. (2019). Les formateurs au travail. Octarès Editions.

Diki-Dikiri, M. (1999). Le signifié et le concept dans la dénomination. Meta, XLIV, 4.

Mayen, P., Ferron, O., Humblot, J-P. et Bazile, J. (2006). Introduire un référentiel de situations dans les référentiels de diplôme en BTS Rapport de recherche de l'unité pour la Direction Générale de l'Enseignement et de la Recherche (DGER). Ministère de l'Agriculture, l'Alimentation et la Forêt. 


\section{REVUE HYBRIDE DE L'ÉDUCATION}

Filliettaz, L., Durand, I. et Trébert, D. (2015). Learning Through Verbal Interactions in the Workplace: The Role and Place of Guidance in Vocational Education and Training. Dans L. Filliettaz et S. Billet (dir.). Francophone perspectives of Learning through work (p. 279-301). Springer.

LeBanc, M. et Tessier, B. (1978). Les étapes de la rééducation. Formalisation et vérification. Criminologie, 11(1), p. 24-45.

Guérin, J. (2012). Activité collective et apprentissages. De l'ergonomie à l'écologie des situations de formation. L'Harmattan.

Jobert, G. (2014). Exister au travail. Les hommes du nucléaire. Érès, coll. Clinique du travail.

Levillain, K. (2015). Les entreprises à mission. Formes, modèle et implications d'un engagement collectif [thèse de doctorat, ParisTech]. Pastel. https://pastel.archives-ouvertes.fr/tel-01178862

Mallet, D., P. Falzon, P. et Vidal-Gomel, C. (2019). La conception participative en simulation : un moyen de développement du "soin de manutention raisonné " [communication orale]. 5e colloque international de didactique professionnelle « Former et développer l'intelligence professionnelle », Université de Sherbrooke, Oct 2019, Longueuil, Canada.

Mayen, P. et Lainé, A. (2015). Travailler avec le vivant. Raison et Passions.

Montlibert (de), C. et Lesne, M. (1972). Formation et analyse sociologique du travail. La Documentation Française.

Montmollin (de), M. (1974). L'analyse du travail préalable à la formation. Presses Universitaires de France.

Montmollin, (de). M. (1990). L'intelligence de la tâche. Presses Universitaires de France.

Mulliez, D. (1998). L'urgence dans l'intervention judiciaire. Cahiers dynamiques, 27, 22-26.

Nachi, M. (2006). Introduction à la sociologie pragmatique. Armand Colin.

Pastré, P. (2006). Apprendre à faire. Dans E. Bourgeois et G. Chapelle (dir.), Apprendre et faire apprendre (p.109-121). Presses Universitaires de France.

Pastré, P. (2011). La didactique professionnelle. Presses Universitaires de France.

Prost, J. et Nojac, E. (2002). Le temps de l'accueil d'urgence. Cahiers dynamiques, 24, 32-36.

Prot, B. (2007a). Un point critique du développement : le concept potentiel. Psychologie de l'interaction, 23-24, 139-169. 


\section{REVUE HYBRIDE DE L'ÉDUCATION}

Prot, B. (2007b). Pour sortir des idées fixes sur l'évaluation. Revue de I'IRES, 3(55), 101-125.

Rogalski, J. et Samurçay, R. (1995). Formation aux activités de gestion d'environnements dynamiques : concepts et méthodes. Education Permanente, (111), 227-242.

Roy, J. et Porchet, E. (2017). Situations critiques : une approche clinique spécifique est nécessaire. Neuropsychiatrie de l'Enfance et de l'Adolescence, 65(6), 338-343.

Santelman, P. (2019). Les opérateurs de formation entre dispersion et inadaptation : un système de formation professionnelle continue peu efficient. Administration \& Éducation, 1(161), 17-22.

Thévenot, L. (1999). L'action comme engagement. Dans CRF (dir.). L'analyse de la singularité de l'action. Presses Universitaires de France.

Tricot, A. et Musial, M. (2020). Précis d'ingénierie pédagogique. De Boeck Supérieur.

Vermersch, P. (1996). L'entretien d'explicitation. ESF.

Vidal-Gomel, Ch. et Rogalski, J. (2007). La conceptualisation et la place des concepts pragmatiques dans l'activité professionnelle et le développement des compétences. @ctivités, 4(1), 49-84, http://www.activites.org/v4n1/v4n1.pdf

Vidal-Gomel, C. (2011). Réflexions et apports théoriques sur la pratique des formateurs et de la simulation. Octarès Editions. 